\title{
Erfahrungsbericht AOTrauma-Deutschland-Reisestipendium 2017
}

Max Daniel Kauther

\author{
Die AOTrauma Deutschland vergibt jährlich mindestens ein Reisestipendium zur \\ Förderung des Erfahrungsaustausches und der Weiterbildung bei angesehenen Ex- \\ perten in unserer Disziplin. Im folgenden Artikel berichtet PD Dr. Max Daniel Kauther, \\ Essen von seinen Erfahrungen in Boston, USA.
}

Durch das großzügige Reisestipendium der AOTrauma Deutschland konnte ich im November 2017 für 4 Wochen das Department of Orthopaedics des Beth Israel Deaconess Medical Center in Boston besuchen. Dieses Level-1 Trauma Center ist Lehrkrankenhaus der Harvard Medical School und war Vorlage für das in meinem Geburtsjahr 1978 veröffentlichte zynische Buch „House of God“, welches für eine Reformierung des medizinischen Ausbildung in den USA in den 80er-Jahren sorgte.

Entgegen der Situation vor 40 Jahren war mein AO-Reisestipendium ein voller Erfolg in einem fachlich und menschlich hervorragenden Team. Die Organisation des Aufenthalts war mit einem eigens für mich zugeschnittenen Rotationsplan überaus professionell und zuvorkommend vorbereitet. So wurde ich dort von den Traumatologen (Professor Edward K. Rodriguez, Professor John J. Wixted und Professor Paul T. Appleton) und den Fußund Sprunggelenkschirurgen (Professor John Y. Kwon und Dr. Christopher P. Miller) kollegial aufgenommen und rundum während der Woche und privat am Wochenende betreut.

Der Tag begann für mich um 7 Uhr mit einer Frühbesprechung mit Tafelvisite und den Fortbildungsveranstaltungen für die Assistenzärzte, in die ich aktiv eingebunden wurde. Hierbei wurde mir die Gelegenheit gegeben, meine sporttraumatologischen Forschungsarbeiten zu präsentieren, welche anschließend vom gesamten Team diskutiert wurden. Zusätzlich gab es die „Grand Rounds“, eine wöchentliche gemeinsame Fortbildungsveranstaltung mit dem Brigham and Women's Hospital mit geladenen Gastrednern. Die Patientenversorgung und Arbeitsorganisation erfolgt durch die Assistenzärzte bereits Stunden vor der Frühbesprechung. Die wöchentliche Arbeitszeit der Assistenzärzte wurde in Boston vor Kurzem auf 80 Stunden begrenzt.

Im OP und den Sprechstunden wurde ich in den Tagesablauf des Teams integriert. So gab es neben dem übli- chen alterstraumatologischen und sporttraumatologischen Spektrum viele spannende, hochkarätige Fälle wie komplexe Becken- und Acetabulumfrakturen, septische Chirurgie mit lappenplastischer Deckung, RingfixateurBehandlungen und Komplexverletzungen des Fußes. Alle Fälle wurden vor- und nachbesprochen und wurden unter Anwendung der AO-Prinzipen unter Beachtung der jeweiligen Weichteilsituation und der Möglichkeit der konservativen Therapie diskutiert. Die konservative Therapie erfolgte mit der Hilfe speziell ausgebildeter Gipstechniker auf hohem Standard.

Die klinisch-operative Versorgung entspricht weitestgehend dem mir aus Deutschland bekannten Vorgehen. Hier sind durch die AOTrauma eine einheitliche Sprache und ein internationaler Standard geschaffen worden. Operative Unterschiede zeigten sich in der häufigeren Anwendung von Allografts sowie der überwiegenden Verwendung von Stahlimplantaten in Boston.

Des Weiteren konnte ich viel über die Facharztausbildung der Orthopädie und Unfallchirurgie in Amerika und Deutschland lernen, welche meiner Ansicht nach besser

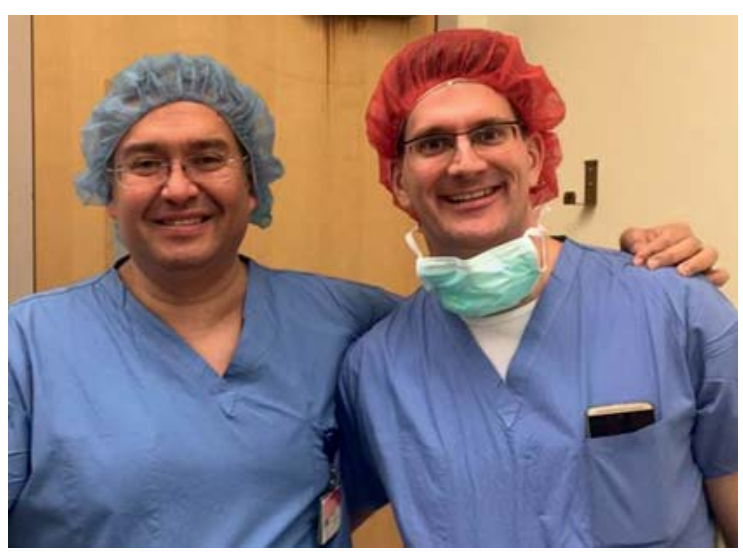

- Abb. 1 Edward K. Rodriguez und Max Daniel Kauther. 


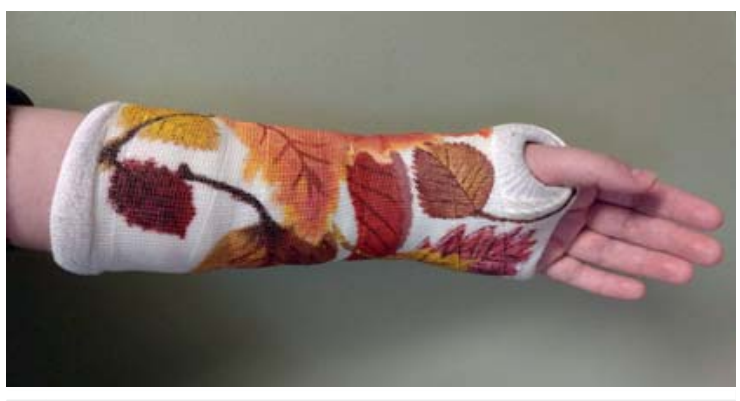

- Abb. 2 Individuelle Verschönerung eines Unterarmgipses.

strukturiert, aber auch fachlich enger aufgestellt ist. Die präklinische Notfallmedizin wird in Amerika komplett durch Sanitäter (paramedics) übernommen und die Intensivmedizin ist nicht wie bei uns integraler Bestandteil der Weiterbildung. Die Schockraumversorgung und Notaufnahme wird von eigenen Fachärzten (emergency medicine) durchgeführt, sodass der Orthopedic Surgeon meist nur zur Versorgung höherwertiger Verletzungen hinzugezogen wird.

Die Lehre in der Facharztausbildung hat in Amerika einen sehr hohen Standard mit täglichen Seminaren vor oder nach der Frühbesprechung. In die studentische Lehre an der Harvard Medical School wurde ich durch Professor Rodriguez als Gastdozent mit eingebunden. Die Gebäude und altehrwürdigen Lehrsäle sind faszinierend.

Des Weiteren konnte ich mit Professor Wixted das muskuloskeletale Forschungslabor der Abteilung besuchen, welches räumlich, apparativ und personell unglaublich gut ausgestattet ist. Der Vertrag der Oberärzte enthält in Boston einen Forschungstag in der Woche, wobei auch hier die Einwerbung von kompetitiven Drittmitteln vergleichbar mit Deutschland erforderlich ist.

Es gab eine von mir so nicht erwartete Offenheit und Wertschätzung gegenüber meiner Meinung zum operativen Vorgehen in komplexen Fällen und ein reges Interesse am deutschen Gesundheits- und Bildungssystem. Hervorheben möchte ich die zeitaufwendige klinische und private Betreuung durch Professor Rodriguez und Professor Wixted. Am Abend und Wochenende wurde ich vom Trauma-Team zur Stadtbesichtigung, Tontaubenschießen, Baseball und Essen mitgenommen - die private Ein-

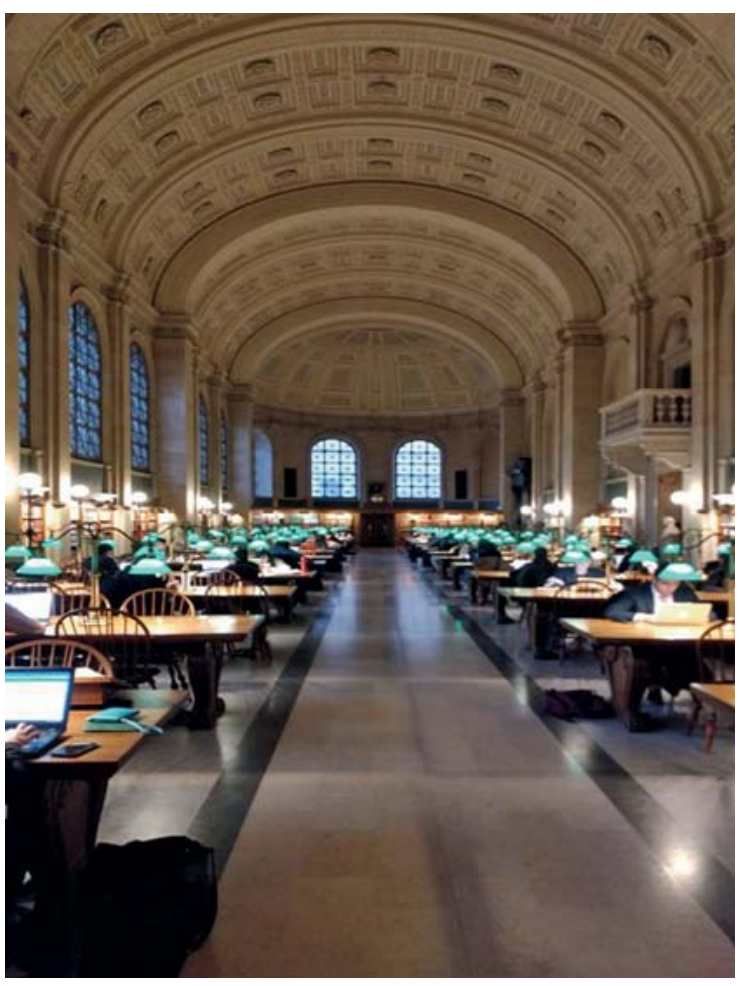

Abb. 3 Lern-Atmosphäre in der Boston Library.

ladung zum Thanksgiving in der Großfamilie von Professor Wixted war ein unvergessliches Ereignis.

Durch das Reisestipendium der AO hatte ich 4 sehr bereichernde Wochen in einem menschlich und fachlich hervorragendem Team, das es geschafft hat, kollegial eine sehr gute Patientenversorgung, Lehre und Forschung mit einer Work-Life-Balance zu verbinden. Für diese Möglichkeit möchte ich mich bei der AOTrauma Deutschland bedanken. Der Gegenbesuch aus Boston zu einem gemeinsamen Trauma-Symposium in Essen ist für 2018 bereits geplant.

PD. Dr. Max Kauther, Universitätsklinikum Essen

E-Mail: maxdaniel.kauther@uk-essen.de

Bibliografie

DOI https://doi.org/10.1055/a-0647-2571

OP-JOURNAL 2018; 34: 182-183 @ Georg Thieme Verlag KG Stuttgart · New York ISSN 0178-1715 